\title{
Obesity is associated with improved survival in community-acquired pneumonia
}

\author{
Anika Singanayagam¹, Aran Singanayagam² and James D. Chalmers ${ }^{3}$
}

\section{Affiliations:}

'Dept of Infection and Immunity, Imperial College, London,

${ }^{2}$ Dept of Respiratory Medicine, Imperial College, London, and

${ }^{3}$ Dept of Respiratory Medicine, University of Dundee, Dundee, UK.

\section{Correspondence:}

A. Singanayagam, Dept of Infection and Immunity, Hammersmith Hospital, Du Cane Road, London, W12 OHS, UK. E-mail: anika.singađagmail.com

ABSTRACT Obesity has been identified as a risk factor for adverse outcomes of 2009 H1N1 influenza. However, the impact of obesity on outcomes of infection remains controversial. There are limited data investigating the effect of obesity on outcomes of community-acquired pneumonia (CAP).

This prospective observational study included patients presenting with CAP who had body mass index (BMI) measured on admission. Outcome measures included 30-day mortality and need for mechanical ventilation or inotropic support (MV/IS).

1079 patients were included, with $21 \%$ classified as obese (BMI $\geqslant 30 \mathrm{~kg} \cdot \mathrm{m}^{-2}$ ). Obesity was independently associated with reduced 30-day mortality from CAP on multivariate analysis (HR 0.53, 95\% CI 0.29-0.98). This was not explained by differences in severity of disease on admission or requirement for MV/IS between obese and nonobese groups. Obese patients had higher median C-reactive protein levels and a higher frequency of sepsis using the systemic inflammatory response syndrome criteria $(72.4 \%$ versus $64.1 \%$; $\mathrm{p}=0.03$ ), than nonobese patients, suggesting greater systemic inflammation.

Obesity was associated with reduced 30-day mortality in patients hospitalised with CAP.

@ERSpublications

Obesity appears to result in improved survival for patients hospitalised with community-acquired pneumonia http://ow.ly/kFUQu 


\section{Introduction}

Community-acquired pneumonia (CAP) is a frequent cause of hospitalisation and a leading cause of mortality worldwide. The mortality rate for patients admitted to hospital with pneumonia is estimated at 5$15 \%$ [1]. CAP is characterised by inflammation of the pulmonary parenchyma in response to an infectious insult, involving systemic and local release of cytokines (tumour necrosis factor (TNF)- $\alpha$, interleukin (IL)-6, IL-8 and IL-1 $\beta$ ) and recruitment of neutrophils. Excessive cytokine production provokes a systemic inflammatory response that can lead to end-organ dysfunction and death [2].

Obesity is a rapidly growing public health problem, associated with an increased risk of poor outcome from many diseases [3]. It has been reported that obesity may be a risk factor for the development of CAP [4]. Some studies, however, have suggested reduced mortality in obese individuals from a variety of diseases, a phenomenon known as the "obesity survival paradox" [5]. The impact of obesity on outcome of infection is complex and remains poorly understood $[6,7]$. Immune function has been shown to be altered in obese individuals [7] and adipokines, secreted from adipose tissue, are known to have pleiotropic effects on the immune system [7].

Obesity has been widely reported as a risk factor for adverse outcome from 2009 H1N1 influenza [8] and, subsequently, the impact of obesity on infectious outcomes has received increasing interest. However, conflicting results have emerged, with adverse outcomes reported in studies of CAP due to 2009 H1N1 influenza [9], but paradoxically, improved outcome in studies of acute bacterial pneumonia unrelated to an influenza pandemic [10-12]. In addition, studies on critically ill patients [13-15] have reported reduced mortality amongst obese patients in the intensive care unit (ICU).

The aim of this study was to investigate the impact of obesity on outcome in a large prospective cohort of patients with CAP.

\section{Methods}

This is a secondary analysis of the Edinburgh pneumonia study dataset, a prospective observational study of patients with CAP, performed in NHS Lothian, Edinburgh, UK, as previously described [16]. The study was approved by the Lothian Research Ethics Committee (UK).

Patients were included in the study if they presented with a new infiltrate on chest radiography and had three or more of the following symptoms or signs: cough, sputum production, breathlessness, pleuritic chest pain or signs consistent with pneumonia on auscultation.

Exclusion criteria were as follows: 1) hospital-acquired pneumonia: development of symptoms $>48 \mathrm{~h}$ following admission or discharge from an acute care facility $<2$ weeks prior to admission; 2) active thoracic malignancy; 3 ) immunosuppression: defined as current, $>28$-day use of oral prednisolone at any dose, or other immunosuppressive drugs (methotrexate, azathioprine, ciclosporin and anti-TNF- $\alpha$ agents), or patients with solid organ transplantation; 4) pulmonary embolism; and 5) patients in whom active treatment was not considered appropriate (palliative care).

Assessment of body mass index

All patients had weight and height measured on admission as part of a nutritional risk assessment score. Body mass index (BMI) was calculated as height/weight ${ }^{2}$.

Patients were classified into recognised BMI subgroups: 1) morbidly obese (BMI $\geqslant 35 \mathrm{~kg} \cdot \mathrm{m}^{-2}$ ); 2) obese (BMI 30- $<35 \mathrm{~kg} \cdot \mathrm{m}^{-2}$ ); 3) overweight (BMI 25-<30 $\mathrm{kg} \cdot \mathrm{m}^{-2}$ ); 4) normal (BMI 18.5-<25 $\mathrm{kg} \cdot \mathrm{m}^{-2}$ ); and 5) underweight $\left(\mathrm{BMI}<18.5 \mathrm{~kg} \cdot \mathrm{m}^{-2}\right)$ as per the World Health Organization guidelines [17].

\section{Data collection and site of care}

At the study sites in NHS Lothian, patients presented either as self-referral to the emergency department or via general practitioner referral to a medical assessment unit. The medical team reviewed patients and made the decision of whether to admit or discharge the patient.

Subsequently, patients spent 12-24 h in the medical assessment unit, from where they were discharged or moved to a specialist ward. Critically ill patients could be admitted at any time to the ICU for invasive ventilation and/or inotropic support, or to the high-dependency unit, which provides intensive monitoring as well as noninvasive ventilation (bi-level or continuous positive airway pressure ventilation), and/or inotropic support. All patients received standard antibiotic therapy in accordance with British Thoracic Society (BTS) guidelines [18].

All patients had baseline observations (pulse, blood pressure, respiratory frequency and temperature) and standard blood tests (full blood count, urea and electrolytes, liver function tests, albumin, coagulation 
profile and C-reactive protein (CRP)) measured on admission. Microbiological testing was left to the discretion of the attending physician, with sputum/blood cultures and Legionella urinary antigen recommended to be sent, according to BTS guidelines. All observations and blood tests were taken within $4 \mathrm{~h}$ of hospital admission.

\section{Severity assessment scores on admission}

On admission, patients were risk assessed using the BTS CURB-65 score (confusion, urea $>7 \mathrm{mmol} \cdot \mathrm{L}^{-1}$, respiratory rate $\geqslant 30$ breaths $\cdot \mathrm{min}^{-1}$, blood pressure $<90 \mathrm{mmHg}$ (systolic) $\leqslant 60 \mathrm{mmHg}$ (diastolic), age $\geqslant 65$ years) [19] and the pneumonia severity index (PSI) [20]. Systemic inflammatory response syndrome (SIRS) criteria (temperature $<36^{\circ} \mathrm{C}$ or $>38^{\circ} \mathrm{C}$, heart rate $>90$ beats $\cdot \mathrm{min}^{-1}$, respiratory rate $\geqslant 20$ breaths $\cdot \mathrm{min}^{-1}$ or arterial carbon dioxide tension $<32 \mathrm{mmHg}$, white blood cells $<4 \times 10^{9}$ cells $\cdot \mathrm{L}^{-1},>12 \times 10^{9}$ cells $\cdot \mathrm{L}^{-1}$ or $>10 \%$ bands) were calculated as previously described [21]. Patients were deemed to have sepsis if they had pneumonia with two or more of the SIRS criteria.

\section{Outcomes}

The primary outcome of interest was 30-day mortality. The secondary outcome was need for mechanical ventilation or inotropic support (MV/IS).

\section{Statistical analysis}

All data were analysed using SPSS (version 13; SPSS Inc., Chicago, IL, USA). Descriptive statistics of demographic and clinical variables are presented as median (interquartile range (IQR)) unless otherwise stated. The Chi-squared test was used to compare categorical data between groups. The t-test was used to compare the mean values of two groups of parametric data, with the Mann-Whitney U-test used to compare two groups of nonparametric data. The Kruskal-Wallis test was used for comparison of more than three groups.

Cox proportional hazards regression analysis was used to adjust for confounders in multivariable analysis. The authors adjusted all models for the PSI [20] and variables not included in the PSI that may impact on survival (prior history of diabetes mellitus, chronic obstructive pulmonary disease (COPD), prior antibiotic treatment, smoking status, multilobar infiltrates and statin use). A sensitivity analysis adjusting for the CURB-65 score as an alternative was also performed [19]. As an alternative method of adjusting for baseline differences between obese and nonobese patients, subjects the authors developed a propensity score from multivariable logistic regression defining the probability of a patient being obese based on their demographic data. The data included in this analysis were age, sex, comorbidities (diabetes mellitus, COPD, liver disease, chronic cardiac disease, cerebrovascular disease and neoplastic disease), current smoking, functional status and nursing home residence, with obesity as the dependent variable. A greedy matching algorithm was used to identify a matched cohort of obese and nonobese individuals with similar propensity. These cohorts were then entered into a Cox proportional hazards model as described above.

Results are expressed as hazard ratios and 95\% confidence intervals. A two-tailed p-value $<0.05$ was considered statistically significant for all analyses.

\section{Results}

This study included 1269 patients with CAP. 1079 patients had available BMI data (190 patients were excluded due to lack of BMI data). Median age was 66 years (IQR 50-78 years). 54\% of the population were aged $>65$ years. There were $473(44 \%)$ patients with a normal body mass index; 335 (31\%) patients were classified as overweight; 203 (19\%) patients were obese; and 22 (2\%) patients morbidly obese. 46 (4\%) patients were classified as underweight. Baseline characteristics of the study population are shown in table 1.

Median duration of admission for the study population was 5 days (IQR 3.0-11.0 days). Duration of admission was highest in the underweight and morbidly obese groups ( 8.0 and 7.5 days, respectively), but no statistically significant difference was seen (table 1).

Diabetes mellitus was more prevalent in patients with obesity (BMI $\geqslant 30 \mathrm{~kg} \cdot \mathrm{m}^{-2}$ ) than normal-weight patients $(14.2 \%$ versus $7.8 \%$; $\mathrm{p}=0.008)$. There was no significant difference in the prevalence of other major comorbidities between obese and nonobese groups. Unequal distribution between BMI groups was noted for patients with COPD $(\mathrm{p}=0.002)$ and in ex-smokers $(\mathrm{p}=0.03)$ (table 1$)$. There was a significant difference in sex distribution among BMI categories $(\mathrm{p}<0.0001)$, with more females in lower BMI groups.

\section{Microbiology}

The rate of positive microbiological diagnosis was low at 30.3\%. The most frequently isolated pathogen was Streptococcus pneumoniae (table 2). The frequency of positive aetiological diagnosis was not significantly 


\begin{tabular}{|c|c|c|c|c|c|c|}
\hline & Morbidly obese & Obese & Overweight & Normal & Underweight & p-value \\
\hline Female & 31.8 & 39.9 & 50.8 & 52.2 & 78.3 & $<0.0001^{*}$ \\
\hline Age years & $62(46-78)$ & $66(52-76)$ & $68(56-78)$ & $66(45-78)$ & 58.5 (39-70) & $0.009 *$ \\
\hline Duration of admission days & $8(4-11)$ & $5(3-11)$ & $5(3-13)$ & $5(2-11)$ & $8(3-13)$ & 0.3 \\
\hline Diabetes mellitus & 13.6 & 14.3 & 11.1 & 7.8 & 6.5 & 0.1 \\
\hline Chronic renal failure & 4.6 & 7.9 & 6.6 & 6.1 & 2.2 & 0.7 \\
\hline Chronic liver disease & 9.1 & 3.9 & 4.2 & 5.7 & 6.5 & 0.7 \\
\hline COPD & 27.3 & 27.1 & 29.3 & 18.2 & 15.2 & $0.002^{*}$ \\
\hline Current smokers & 18.2 & 35.0 & 36.7 & 32.0 & 39.1 & 0.3 \\
\hline Ex-smokers & 45.5 & 23.2 & 21.8 & 21.6 & 10.9 & $0.03^{*}$ \\
\hline
\end{tabular}

Data presented as $\mathrm{n}(\%)$, median (interquartile range) or \%, unless otherwise stated. COPD: chronic obstructive pulmonary disease. ${ }^{*}$ : $<<0.05$.

different between the groups (morbidly obese $36.4 \%$, obese $30.0 \%$, overweight $28.7 \%$, normal $31.1 \%$ and underweight $32.6 \% ; \mathrm{p}=0.9$ ). There were no significant differences in the comparisons of individual organisms between the groups, although numbers were small for many isolates.

\section{Severity assessment and inflammatory response on admission}

There was no difference in severity of illness upon admission between obese and normal groups (35.5\% versus $29.6 \% ; \mathrm{p}=0.2$ with CURB-65 score $\geqslant 3$ ) or across any of the BMI groups (table 3 ).

Obese patients were more likely to be classified as having sepsis according to the SIRS criteria compared to patients with normal BMI $(\mathrm{p}=0.002)$, and comparison across all BMI groups showed a significant association with sepsis criteria indicating a higher frequency of sepsis in obese and overweight individuals (table 3). Median CRP was higher in obese patients than normal-weight patients $\left(186 \mathrm{mg} \cdot \mathrm{L}^{-1}\right.$ versus $\left.151 \mathrm{mg} \cdot \mathrm{L}^{-1}\right)$ and a significant association was seen across BMI groups, indicating higher CRP in the obese group (table 3). There was no difference in albumin levels between any of the BMI groups.

\section{The relationship between $\mathrm{BMI}$ and outcomes}

There was no difference in immediate requirement for mechanical ventilation or vasopressor support between patients with obesity $\left(\mathrm{BMI}>30 \mathrm{~kg} \cdot \mathrm{m}^{-2}\right)$ and nonobese patients $(8.9 \%$ versus $7.0 \% ; \mathrm{p}=0.3)$, or across any of the BMI groups (table 3 ).

The 30 -day mortality rate was $9.6 \%$. The mortality rate in the obese group was $7.4 \%, 10.5 \%$ in the overweight group and $11 \%$ in patients with a normal BMI. There were few patients in the morbidly obese and underweight groups. On univariate analysis, the difference in 30-day mortality between patients with

\section{TABLE 2 Microbiology of the study population}

$\begin{array}{lc}\text { Patients with a microbiological diagnosis } & 327(30.3) \\ \text { Streptococcus pneumoniae } & 186(56.9) \\ \text { Haemophilus influenzae } & 33(10.1) \\ \text { Legionella pneumophila } & 12(3.7) \\ \text { Methicillin-sensitive Staphylococcus aureus } & 28(8.6) \\ \text { Methicillin-resistant S. aureus } & 2(0.6) \\ \text { Mycoplasma pneumoniae } & 19(5.8) \\ \text { Respiratory viruses } & 22(6.7) \\ \text { Chlamydophila pneumoniae } & 1(0.3) \\ \text { Gram-negative Enterobacteriaceae } & 10(3.1) \\ \text { Pseudomonas aeruginosa } & 2(0.6) \\ \text { Stenotrophomonas maltophilia } & 1(0.3) \\ \text { Other bacteria } & 11(3.4)\end{array}$

Data are presented as $\mathrm{n}(\%)$. 
TABLE 3 Severity markers according to body mass index class

\begin{tabular}{|c|c|c|c|c|c|c|}
\hline & Morbidly obese & Obese & Overweight & Normal & Underweight & p-value \\
\hline Subjects & $22(2.0)$ & 203 (18.9) & $335(31.1)$ & $473(43.8)$ & $46(4.3)$ & \\
\hline \multicolumn{7}{|l|}{ PSI class } \\
\hline$|-| \mid$ & $9(40.9)$ & $69(34.0)$ & 115 (34.3) & $157(33.2)$ & 17 (37.0) & 0.9 \\
\hline III & $1(4.5)$ & 33 (16.3) & $54(16.1)$ & $96(20.3)$ & 13 (28.3) & 0.07 \\
\hline IV & $9(40.9)$ & $65(32.0)$ & $108(32.2)$ & $141(29.8)$ & 13 (28.3) & 0.8 \\
\hline V & $3(13.6)$ & $36(17.7)$ & $58(17.3)$ & $79(16.7)$ & $3(6.5)$ & 0.4 \\
\hline \multicolumn{7}{|l|}{ CURB-65 class } \\
\hline $0-1$ & $8(36.4)$ & 81 (39.9) & $135(40.3)$ & $204(43.1)$ & 23 (50.0) & 0.6 \\
\hline 2 & $6(27.3)$ & $50(24.6)$ & $83(24.8)$ & $129(27.3)$ & $10(21.7)$ & 0.9 \\
\hline $3-5$ & $8(36.4)$ & 72 (35.5) & $117(34.9)$ & $140(29.6)$ & 13 (28.3) & 0.7 \\
\hline Serum albumin $\mathbf{g} \cdot \mathrm{L}^{-1}$ & $38(35-41)$ & $35(33-40)$ & 37 (32-39) & $37(33-40)$ & $36(33-38)$ & 0.1 \\
\hline C-reactive protein $\mathrm{mg} \cdot \mathrm{L}^{-1}$ & $94(16-268)$ & $186(76-316)$ & $165(61-266)$ & $151(55-300)$ & 105 (27-207) & $0.04^{*}$ \\
\hline Sepsis criteria" & $13(59.1)$ & $149(73.4)$ & $229(68.4)$ & $289(61.1)$ & $30(65.2)$ & $0.02 *$ \\
\hline Time to clinical stability days & $4(2-5)$ & $4(2-7)$ & $4(2-7)$ & $4(2-7)$ & $5(2-8)$ & 0.8 \\
\hline Requirement for MV/IS & $1(4.6)$ & $19(9.4)$ & $19(5.7)$ & $38(8.0)$ & $3(6.5)$ & 0.5 \\
\hline
\end{tabular}

Data are presented as $\mathrm{n}(\%)$ or median (interquartile range). PSI: pneumonia severity index; CURB-65: confusion, urea $>7 \mathrm{mmol} \cdot \mathrm{L}^{-1}, \mathrm{respiratory}$ rate $\geqslant 30$ breaths $\cdot \mathrm{min}^{-1}$, blood pressure $<90 \mathrm{mmHg}$ (systolic) $\leqslant 60 \mathrm{mmHg}$ (diastolic), age $\geqslant 65$ years; MV/IS: mechanical ventilation or inotropic support. " : presence of two or more systemic inflammatory response syndrome criteria. *: $p<0.05$.

obesity $\left(B M I \geqslant 30 \mathrm{~kg} \cdot \mathrm{m}^{-2}\right)$ and nonobese $\left(B M I<30 \mathrm{~kg} \cdot \mathrm{m}^{-2}\right)$ patients $(6.7 \%$ versus $10.3 \% ; \mathrm{p}=0.3)$ and obesity $\left(\mathrm{BMI}>30 \mathrm{~kg} \cdot \mathrm{m}^{-2}\right)$ versus normal weight $(6.7 \%$ versus $11.0 \% ; \mathrm{p}=0.07)$ failed to reach significance.

Using Cox proportional hazards regression, adjusting for PSI and other confounders not included in the PSI, obesity was independently associated with reduced 30-day mortality (HR 0.53, 95\% CI 0.29-0.98). The hazard ratio for overweight compared to normal BMI was 0.90 (95\% CI 0.78-1.04). The analysis for underweight was limited by the low number of patients and events, and an adequate model could not be constructed comparing morbidly obese patients against the other groups as there were only 22 patients in this subcategory. Sensitivity analyses adjusting for CURB-65 instead of PSI gave very similar results to the main analysis (table 4).

In the propensity-adjusted analysis, the relationship between obesity and reduced mortality persisted (HR 0.50, 95\% CI 0.25-0.99). Entering BMI as a continuous variable into the model, increasing BMI was independently associated with reduced 30-day mortality (HR 0.93, 95\% CI 0.89-0.97).

\section{BMI and outcome in critically ill patients}

We further sought to identify whether there was any difference in impact of obesity on outcome in patients who were critically ill (requiring MV/IS on admission) and those who were not critically ill. This analysis focussed on the comparison between obese $\left(B M I \geqslant 30 \mathrm{~kg} \cdot \mathrm{m}^{-2}\right.$ ) and nonobese $\left(B M I<30 \mathrm{~kg} \cdot \mathrm{m}^{-2}\right.$ ) groups because of the significant differences noted above.

Among critically ill patients (those requiring MV/IS), there was no difference in 30-day mortality rate $(50.0 \%$ versus $45.0 \% ; \mathrm{p}=0.7)$ between obese and nonobese groups (table 5$)$.

Among those patients who did not require MV/IS on admission, the 30-day mortality rate was significantly lower among obese patients than nonobese patients $(2.4 \%$ versus $7.7 \% ; \mathrm{p}=0.007)$. This was despite no

TABLE 4 Cox proportional hazards regression analysis showing the relationship between body mass index (BMI) and 30-day mortality

BMI

Underweight
Normal
Overweight
Obese

PSI: pneumonia severity index; CURB-65: confusion, urea $>7 \mathrm{mmol} \cdot \mathrm{L}^{-1}$, respiratory rate $\geqslant 30 \mathrm{breaths} \cdot \mathrm{min}^{-1}$, blood pressure $<90 \mathrm{mmHg}$ (systolic) $\leqslant 60 \mathrm{mmHg}$ (diastolic), age $\geqslant 65$ years.

$\begin{array}{cc}0.74(0.26-2.06) & 0.79(0.31-1.98) \\ 1.0 \text { (reference) } & 1.0 \text { (reference) } \\ 0.90(0.78-1.04) & 0.83(0.54-1.29) \\ 0.53(0.29-0.98) & 0.57(0.32-1.01)\end{array}$

\section{PSI-adjusted HR $(95 \% \mathrm{Cl}) \quad$ CURB-65-adjusted HR $(95 \% \mathrm{Cl})$}


TABLE 5 Comparison between those requiring and not requiring mechanical ventilation or inotropic support

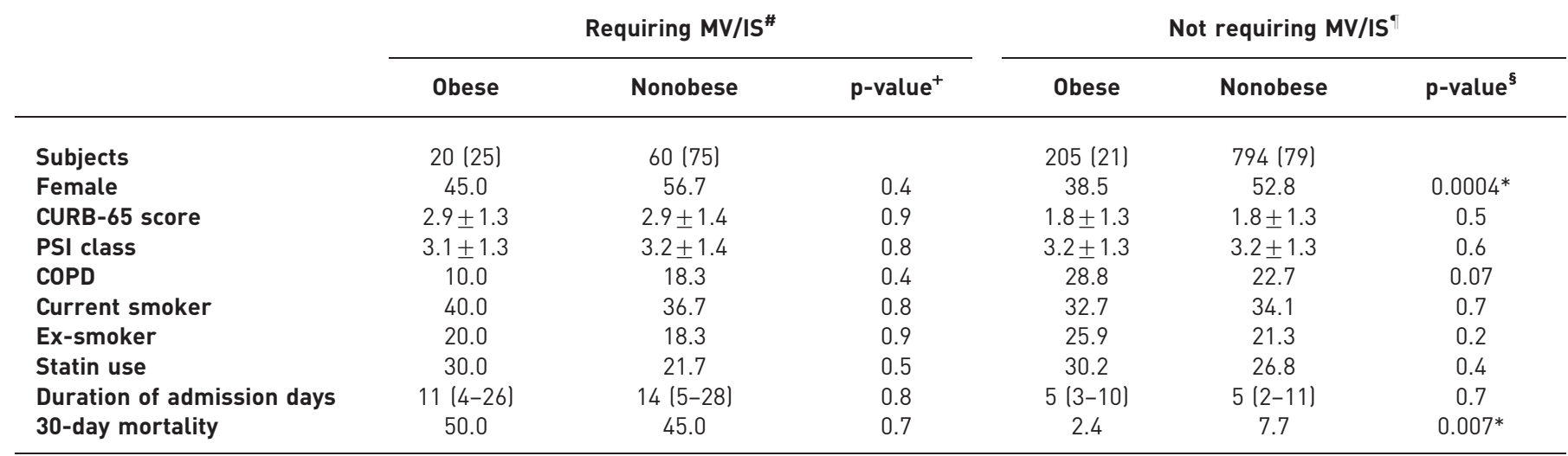

Data are presented as $\mathrm{n}(\%), \%$, mean \pm SD or median (interquartile range), unless otherwise stated. MV/IS: mechanical ventilation or inotropic support; CURB-65: confusion, urea $>7 \mathrm{mmol} \cdot \mathrm{L}^{-1}$, respiratory rate $\geqslant 30$ breaths $\cdot \mathrm{min}^{-1}$, blood pressure $<90 \mathrm{mmHg}$ (systolic) $\leqslant 60 \mathrm{mmHg}$ (diastolic), age $\geqslant 65$ years; PSI: pneumonia severity index; COPD: chronic obstructive pulmonary disease. ${ }^{\#}: \mathrm{n}=80 ;{ }^{\circ}: \mathrm{n}=999 ;{ }^{+}:$comparing obese and nonobese patients requiring MV/IS; ${ }^{\S}$ : comparing obese and nonobese patients not requiring MV/IS. *: $p<0.05$.

difference in mean CURB-65 score, mean PSI class or COPD status between this group of "non-critically ill" obese and nonobese patients (see table 5).

\section{Discussion}

Our study shows that obesity $\left(\mathrm{BMI} \geqslant 30 \mathrm{~kg} \cdot \mathrm{m}^{-2}\right.$ ) is independently associated with reduced 30-day mortality from CAP. This was despite no difference in disease severity on admission and no difference in immediate requirement for higher-level care input (MV/IS) between obese and nonobese groups.

These results are intriguing. Obese patients had higher levels of CRP and a higher frequency of sepsis using the SIRS criteria, suggesting greater systemic inflammation, yet had a significantly lower 30-day mortality. It has been hypothesised that improved outcomes in obese individuals may be due to immunomodulation in critically ill patients. In contrast, however, our results suggest that the improved outcomes are in noncritically ill patients with CAP. The reasons for these findings are unclear.

Our results extend the findings of a recent study by CoRrales-Medina et al. [10] of 266 hospitalised patients with community-acquired bacterial pneumonia. Our study, which includes a larger sample size and is prospective in design, concurs with the findings from this smaller study. CoRRALES-Medina et al. [10] showed that increasing BMI values were associated with reduced 30-day mortality (OR 0.88, 95\% CI $0.81-$ $0.96 ; \mathrm{p}<0.01$ ) on multivariate analysis. Furthermore, in two large cohort studies of pneumonia mortality, higher BMI was again associated with reduced mortality. The study of 2600 males by LACrOIX et al. [12] showed that the risk of pneumonia mortality was 2.6 times higher in males in the lowest BMI quartile compared to the highest quartile. INOUE et al. [11] demonstrated BMI $>25 \mathrm{~kg} \cdot \mathrm{m}^{-2}$ to be a protective factor against pneumonia mortality in a cohort study of 110000 Japanese adults (HR 0.7, 95\% CI 0.5-0.8; $\mathrm{p}<0.001$ ). These studies used International Classification of Diseases- 9 codes to classify CAP, which has previously been suggested to miss around $25 \%$ of hospital admissions with CAP [22]. Furthermore, these studies may have mistakenly included patients with conditions presenting similarly to CAP (such as influenza, upper respiratory tract infections or heart failure), which are known to have distinct outcomes in obese patients. In contrast, inclusion criteria for our study required clinical and radiographic confirmation of CAP, improving the validity of our results.

This paradoxical protective effect of obesity on mortality has been reported in other respiratory illnesses, such as acute exacerbations of COPD [23], as well as ICU outcome studies [13, 24], including post-surgical patients with acute respiratory distress syndrome [14] and a subset of ICU patients with acute lung injury [15]. Conflicting results do exist, and a meta-analysis of ICU mortality studies by HoGUE et al. [25] concluded that obesity was not associated with increased risk for ICU mortality, but may have been associated with lower hospital mortality after critical illness.

Obesity is likely to have a complex interplay with pneumonia outcome. Obesity is associated with increased total mortality rates in the general population and increased incidence of comorbidities such as diabetes mellitus, gastro-oesophageal reflux disease and hypertension [26]. Furthermore, obese patients are thought 
of as a clinical management challenge associated with impaired lung mechanics and altered pharmacokinetics, as well as nursing and rehabilitation difficulties. Obesity appears to increase the risk of pneumonia in general population [4]. The suggestion of a protective effect of obesity on pneumonia mortality therefore appears incongruous.

One potential impacting factor is the alteration of pulmonary immune responses in the obese [27]. Obesity is associated with a chronic inflammatory state $[7,27,28]$, leading to speculation that this might lead to a heightened state of host defence [7]. Studies have identified that adipokines, secreted from adipose tissue, have pro- and anti-inflammatory effects [7]. One such adipokine, leptin, is seen in increased levels in obese individuals [29]. The role of leptin in the response to sepsis and pulmonary infections remains a subject of debate. Mouse models of leptin deficiency ( $o b / o b$ mice) have exhibited reduced bacterial clearance and increased mortality, shown to be related to impaired alveolar macrophage phagocytosis, reduced lung neutrophil counts and levels of pro-inflammatory cytokines [30, 31]. Exogenous administration of leptin improved survival and pulmonary bacterial clearance and reduced bacteraemia in ob/ob mice [31].

Alternative explanations for our findings might be that obese patients are referred more readily for intensive management strategies, or that they are more likely to be admitted to hospital, even with milder disease. However, we showed no significant difference in admission CURB-65 or PSI scores for the two groups, indicating that this does not explain the findings observed in our study. These scores are imperfect markers of severity and, therefore, the possibility of confounding by severity cannot be excluded. Despite having strict radiological inclusion criteria for our study, it is possible that CAP may have been misdiagnosed in obese patients. In particular, radiological changes can be difficult to interpret in obese patients [32]. Finally, the pharmacokinetics of antibiotics are altered in obese patients and this may contribute to an altered response to antibiotic therapy [33].

The frequency of positive microbiology testing was low in this study and it was not possible to demonstrate any differences between groups in the frequency of causative organisms. This could be considered a limitation of our study. We did not measure other inflammatory markers such as TNF- $\alpha$, IL- 6 or leptin levels, which might have provided additional useful information. Lastly, BMI has its own limitations in defining obesity and it has been suggested that alternative measurements such as lean body mass or waist:hip ratio might be of greater value. Future studies should assess the correlation of more accurate measures of body mass with outcome in CAP.

In conclusion, we have shown that obese and nonobese patients present to hospital with CAP of similar severity. However, obese patients have a significantly improved survival compared to their nonobese counterparts. In our understanding of the interplay between obesity and pulmonary infection, many questions remain unanswered. Our study adds to the debate on the "obesity paradox" and highlights our lack of understanding of the implications of obesity in areas such as immunomodulation, pharmacokinetics and lung mechanics. Further clinical and mechanistic studies in this important area are warranted.

\section{References}

Singanayagam A, Singanayagam A, Elder DHJ, et al. Is community-acquired pneumonia an independent risk factor for cardiovascular disease? Eur Respir J 2012; 39: 187-196.

2 Delclaux C, Azoulay E. Inflammatory response to infectious pulmonary injury. Eur Respir J 2003; 22: Suppl. 42, $10 \mathrm{~s}-14 \mathrm{~s}$.

3 World Health Organization: Obesity and Overweight: Fact Sheet No 311. www.who.int/mediacentre/factsheets/ fs311/en/ Date last updated: March 2013. Date last accessed: May 5, 2013.

4 Baik I, Curhan GC, Rimm EB, et al. A prospective study of age and lifestyle factors in relation to communityacquired pneumonia in US men and women. Arch Intern Med 2000; 160: 3082-3088.

Morse S, Gulati R, Reisin E. The obesity paradox and cardiovascular disease. Curr Hypertens Rep 2010; 12: 120-126. Karlsson EA, Beck MA. The burden of obesity on infectious disease. Exp Biol Med (Maywood) 2010; 235: 1412-1424. Ouchi N, Parker JL, Lugus JJ, et al. Adipokines in inflammation and metabolic disease. Nat Rev Immunol 2011; 11: 85-97.

8 Fezeu L, Julia C, Henegar A, et al. Obesity is associated with higher risk of intensive care unit admission and death in influenza A (H1N1) patients: a systematic review and meta-analysis. Obes Rev 2011; 12: 653-659.

9 Riquelme R, Jiménez P, Videla AJ, et al. Predicting mortality in hospitalized patients with 2009 H1N1 influenza pneumonia. Int J Tuberc Lung Dis 2011; 15: 542-546.

10 Corrales-Medina VF, Valayam J, Serpa JA, et al. The obesity paradox in community-acquired bacterial pneumonia. Int J Infect Dis 2011; 15: e54-e57.

11 Inoue Y, Koizumi A, Wada Y, et al. Risk and protective factors related to mortality from pneumonia among middleaged and elderly community residents: the JACC Study. J Epidemiol 2007; 17: 194-202.

12 LaCroix AZ, Lipson S, Miles TP, et al. Prospective study of pneumonia hospitalizations and mortality of US older people: the role of chronic conditions, health behaviors, and nutritional status. Public Health Rep 1989; 104: 350-360.

13 Oliveros H, Villamor E. Obesity and mortality in critically ill adults: a systematic review and meta-analysis. Obesity (Silver Spring) 2008; 16: 515-521.

14 Memtsoudis SG, Bombardieri AM, Ma Y, et al. Mortality of patients with respiratory insufficiency and adult respiratory distress syndrome after surgery: the obesity paradox. J Intensive Care Med 2012; 27: 306-311. 

mechanically ventilated adults with acute lung injury. Crit Care Med 2006; 34: 738-744.

16 Chalmers JD, Singanayagam A, Murray MP, et al. Prior statin use is associated with improved outcomes in community-acquired pneumonia. Am J Med 2008; 121: 1002-1007.

17 World Health Organisation. Physical Status: the Use and Interpretation of Anthropometry. Report of a WHO Expert Committee. WHO Technical Report; Series 854. Geneva, World Health Organization, 1995.

18 British Thoracic Society Standards of Care Committee. BTS guidelines for the management of community acquired pneumonia in adults. Thorax 2001; 56: Suppl. 4, iv1-iv64.

19 Lim WS, van der Eerden MM, Laing R, et al. Defining community acquired pneumonia severity on presentation to hospital: an international derivation and validation study. Thorax 2003; 58: 377-382.

20 Fine MJ, Auble TE, Yealy DM, et al. A prediction rule to identify low-risk patients with community-acquired pneumonia. N Engl J Med 1997; 336: 243-250.

21 Bone RC, Balk RA, Cerra FB, et al. Definitions for sepsis and organ failure and guidelines for the use of innovative therapies in sepsis. The ACCP/SCCM Consensus Conference Committee. American College of Chest Physicians/ Society of Critical Care Medicine. Chest 1992; 101: 1644-1655.

22 van de Garde EM, Oosterheert JJ, Bonten M, et al. International classification of diseases codes showed modest sensitivity for detecting community-acquired pneumonia. J Clin Epidemiol 2007; 60: 834-838.

23 Lainscak M, von Haehling S, Doehner W, et al. Body mass index and prognosis in patients hospitalized with acute exacerbation of chronic obstructive pulmonary disease. J Cachexia Sarcopenia Muscle 2011; 2: 81-86.

24 Tremblay A, Bandi V. Impact of body mass index on outcomes following critical care. Chest 2003; 123 : $1202-1207$.

25 Hogue CW Jr, Stearns JD, Colantuoni E, et al. The impact of obesity on outcomes after critical illness: a metaanalysis. Intensive Care Med 2009; 35: 1152-1170.

26 Solomon CG, Manson JE. Obesity and mortality: a review of the epidemiologic data. Am J Clin Nutr 1997; 66: Suppl. 4, 1044S-1050S.

27 Mancuso P. Obesity and lung inflammation. J Appl Physiol 2010; 108: 722-728.

28 Florez H, Castillo-Florez S, Mendez A, et al. C-reactive protein is elevated in obese patients with the metabolic syndrome. Diabetes Res Clin Pract 2006; 71: 92-100.

29 Considine RV, Sinha MK, Heiman ML, et al. Serum immunoreactive-leptin concentrations in normal-weight and obese humans. N Engl J Med 1996; 334: 292-295.

30 Wieland CW, Stegenga ME, Florquin S, et al. Leptin and host defense against Gram-positive and Gram-negative pneumonia in mice. Shock 2006; 25: 414-419.

31 Hsu A, Aronoff DM, Phipps J, et al. Leptin improves pulmonary bacterial clearance and survival in ob/ob mice during pneumococcal pneumonia. Clin Exp Immunol 2007; 150: 332-339.

32 Uppot RN, Sahani DV, Hahn PF, et al. Effect of obesity on image quality: fifteen-year longitudinal study for evaluation of dictated radiology reports. Radiology 2006; 240: 435-439.

33 Pai MP, Bearden DT. Antimicrobial dosing considerations in obese adult patients. Pharmacotherapy 2007; 27: 1081-1091. 